Jull, G. and Hall, T. 2018. Cervical musculoskeletal dysfunction in headache: How should it be defined? Musculoskeletal Science and Practice. 38: pp. 148-150.

\title{
Cervical musculoskeletal dysfunction in headache: how should it be defined?
}

\author{
Gwendolen Jull, Toby Hall
}

Gwendolen Jull MPhty, PhD, FACP

Emeritus Professor, Physiotherapy

School of Health and Rehabilitation Sciences

The University of Queensland

Australia

g.jull@uq.edu.au

Toby Hall MSc, PhD, FACP

Adjunct Associate Professor

School of Physiotherapy and Exercise Science

Curtin University

Australia

halltm@netspace.net.au 


\section{Highlights}

- Neck pain frequently accompanies migraine and tension-type headaches

- There is not consensus on the cause of this headache related neck pain

- Research into a cervical cause should seek a pattern of related dysfunction 


\begin{abstract}
Currently, the literature is divided about the role of cervical musculoskeletal dysfunction as the cause of migraine and tension-type headache related neck pain. Clarification is essential for both hypotheses on the pathogenesis of these headaches and their variants and for decisions on the suitability of local neck treatments, both from research and clinical practice perspectives. Reasons for the disparate findings could relate to participant selection in headache studies and/or the bases on which decisions on the presence of cervical musculoskeletal dysfunction are reached. Propositions towards gaining a clearer picture of migraine and tension-type headache related neck pain include first, stricter inclusion criteria and reporting of headache characteristics of study participants. Second, reliance on pain sensitivity or the presence of neck tenderness/trigger points as measures be discarded, as they are not uniquely tied to a musculoskeletal disorder. Instead, reliance is placed on tests of musculoskeletal (dys)function. Third, the values and interpretation of single measures or tests of impairment/dysfunctions can be non-informative and do not reflect the presentation of cervical musculoskeletal disorders. Rather, a typical presentation includes at a fundamental level, interrelated changes in cervical movement, segmental joint and muscle function. We advocate that these measures be adopted as the core set of related measures to define cervical musculoskeletal dysfunction in headache. This does not deter inclusion of other measures of interest or qualification.
\end{abstract}

Key words: headache, neck pain, diagnosis 
Recurrent headaches such as migraine and tension-type headache (TTH) are common disorders resulting in considerable personal, social and economic costs. ${ }^{12}$ Understandably, patients, clinicians and researchers alike seek treatments that may relieve headache symptoms or triggers. In recent years, there has been renewed interest and research into the role of neck pain in migraine and TTH. Neck pain frequently accompanies these headaches, reflecting the interaction between trigeminal and cervical afferents in the trigeminocervical nucleus. ${ }^{3}$ The interesting question relates to the origin or cause of the neck pain. It is argued variously (i) that neck pain is part of the migraine symptomology and not related to cervical musculoskeletal dysfunction, ${ }^{45}$ (ii) that the neck pain is related to a cervical musculoskeletal disorder and an integral feature of migraine ${ }^{67}$ or (iii) that neck pain is a common secondary consequence of headache. ${ }^{8}$ It is important for future research to clarify the role of cervical musculoskeletal dysfunction in these headache types. It has central implications for hypotheses on the pathogenesis of migraine and TTH as well as the variants of these headache types. It has particular significance for decisions on the suitability of local neck treatments such as manipulative therapy and therapeutic exercise for these headache forms.

\section{Challenges}

Reflecting current opinions, literature examining cervical musculoskeletal dysfunction in migraine and TTH presents a confusing picture. Some studies report findings of cervical musculoskeletal dysfunction in migraine and TTH compared to non-headache groups, others do not. ${ }^{69-13}$ Further research is vital to resolve these discrepancies to guide both clinical practice and future clinical trials. We suggest that there are two major factors that must be addressed in future research to define a possible causative, contributing, comorbid or no role of cervical musculoskeletal dysfunction in migraine and TTH. The first is to ensure the participant has the headache relevant to the research question. The second is in establishing fundamental criteria which define the presence of cervical musculoskeletal dysfunction.

\section{Population selection}

The clinical presentations of recurrent migraine or TTH are various which has implications for participant selection in research into the role of cervical musculoskeletal dysfunction. At a first level, migraine and TTH may be episodic or chronic. The International Classification of Headache Disorders' (ICHD_3) definition of chronic migraine is that the patient has 15 or more headache days per month with at least eight of these having the features of migraine. ${ }^{14}$ This means that seven of 15 headaches (or more if headache frequency is greater) could be other 
types, e.g. 'TTH-like' or possibly cervicogenic headache which can have a migraine-like presentation. ${ }^{15}$ If cervicogenic, any conclusion about the association of musculoskeletal dysfunction with chronic migraine would be confounded and an accurate conclusion could not be made about a relationship of neck dysfunction to migraine. The situation is similar for chronic TTH where some headaches may be migraine-like or possibly other forms. Even when a person reports a single headache form, symptom overlap and mixed headaches are not uncommon which challenge diagnosis. ${ }^{16}$ Headache is often diagnosed by predominant features even though features of another headache may be present. ${ }^{14} \mathrm{We}$ contend that these challenges in diagnosis need to be addressed in future research design if we are to have a clear picture of the role of cervical musculoskeletal dysfunction in headaches as migraine and TTH and their various presentations.

If the question is whether cervical musculoskeletal dysfunction is the cause of the neck pain accompanying migraine or TTH, then participants who have the typical form of the headache must be recruited to provide assurity of outcomes devoid of possible confounding diagnostic variables. The question of whether cervical musculoskeletal dysfunction is the cause of neck pain in migraine and TTH cannot be answered if participants have chronic headache forms as defined in the ICHD_3 or atypical or mixed forms of these headaches. The role of cervical musculoskeletal dysfunction in these latter headache forms needs to be researched as separate, important questions. It is only through such precise research will the relationship between cervical musculoskeletal dysfunction and migraine and TTH related neck pain be fully understood.

In addressing the challenge of participant selection, participants are usually included into a study based on a medical diagnosis and/or a statement that headaches fulfilled the ICHD_3 classification criteria for migraine or TTH as relevant. This is a sound beginning but requires the reader to have a degree of faith rather than absolute assurity of accurate participant selection. Confidence that the study contained participants with the headache of interest could be strengthened if researchers were required to provide additional information about the headaches, aside from frequency, intensity and duration as part of participant demographics. This could include a summary description of the characteristics and temporal pattern of participants' headache to demonstrate that they met the criteria for a typical headache. When the research question relates to potential cervical musculoskeletal dysfunction in chronic migraine or TTH, it would be clearer if participant demographics included information on the 
frequencies of both the migraine and non-migrainous headaches as well the latter's classification(s). Any person where 'other' headaches included cervicogenic headaches should be excluded from the study.

It is likewise important to consider and report participant recruitment sources. Participants recruited from the community by advertisement may differ from those recruited from either neurology practices or a tertiary headache clinic. Participants from the latter source may have more severe forms of the headache disorder or a higher incidence of biological or psychological comorbidities. Recruitment strategies could introduce a recruitment bias into the incidence of cervical musculoskeletal dysfunction and interpretation of results. Research directly investigating this issue is warranted.

In summary, only persons who have the classical features of the headache should be recruited to make clear and accurate conclusions on the presence or association of cervical musculoskeletal dysfunction with migraine or TTH. The role of any cervical musculoskeletal dysfunction in persons classified as probable migraine or TTH (ie. headaches classifiable as migraine or TTH but containing other features of other headaches) on ICHD_3 criteria or in persons with chronic migraine or TTH are separate and necessary questions. Accurate recruitment is a vital factor if we are to gain an true understanding of the relationship of cervical musculoskeletal dysfunction to neck pain in these headache types and their variants.

\section{Measuring cervical musculoskeletal dysfunction}

It is not possible to identify reliably, the possible contribution of the neck to headache with any imaging or laboratory test. Reliance is on clinical tests. The choice of relevant tests, their application and interpretation are vital to valid conclusions about the relationship of any headache associated neck pain to a local cervical condition.

\section{Choosing musculoskeletal tests}

Tests chosen should uniquely define a cervical musculoskeletal disorder. For example, interpreting trigger points as a primary musculoskeletal dysfunction is questionable as they are found in TTH, migraine and cervicogenic headache ${ }^{17} 18$ as well as in association with visceral disease. ${ }^{19}{ }^{20}$ Likewise relying on tests of pain sensitivity such as palpation of tenderness of cervical structures (muscle or bone) or reproduction of headache as an indicator of local cervical causes of neck pain ${ }^{7}$ are not absolute signs of cervical musculoskeletal dysfunction. 
They may just be reflective of a local nociceptive provocation of a centrally sensitised trigeminocervical nucleus, which presents in both migraine and TTH.

Tests of function (i.e. functional integrity of cervical articular, nervous, neuromuscular and sensorimotor systems) stand to be more conclusive than tests of pain sensitivity. Many different clinical tests are used or advocated to investigate cervical musculoskeletal dysfunction in migraine and TTH headache. A recent consensus report by headache experts in physiotherapy advocated 11 tests of which nine related to function and two to pain sensitivity. ${ }^{21}$ A subsequent study investigating these tests in a migraine cohort ${ }^{6}$ found that four of the nine functional tests were significantly different in the migraine group compared to the control group. Five of the functional tests were not significantly different, which could suggest they are not core features which define cervical musculoskeletal dysfunction. Surprisingly one was range of cervical movement as reduced motion is a fundamental feature of cervical musculoskeletal dysfunction. ${ }^{22}$ It could also illustrated that single tests or interpretation of tests in a singular way is often of limited value. There is large inter-individual variation in range of cervical movement at any age and it declines with age ${ }^{23}$ which makes it difficult to nominate a particular cut off value for an individual. Similarly, while cervical flexor and extensor weakness is a feature of a cervical disorder, a single measure of cervical muscle strength is also subject to gender and age effects, meaning that there are usually no definitive cut off values of diagnostic usefulness for an individual. Additionally, not all impairments will be present in every patient, e.g. neck pain may or may not be associated with a forward head posture.

As is well appreciated in clinical practice, musculoskeletal disorders are characterised by a pattern of dysfunction, not an isolated or singular sign. We contend that to make a decision that migraine or TTH associated neck pain is caused by cervical musculoskeletal dysfunction, then it should be associated with a pattern of dysfunction, not isolated or unrelated measures. This is not a novel suggestion as studies have already demonstrated the strength of a pattern of findings to diagnose a cervical disorder. ${ }^{12} 24$ Appropriate statistical analysis should be undertaken which seeks a pattern of dysfunction linked to the neck pain rather than just investigate between group differences in randomly chosen physical tests.

The central question is what physically impaired functions constitute a fundament pattern characteristic of a musculoskeletal disorder which should be included in each study to claim cervical musculoskeletal dysfunction is the cause of neck pain accompany migraine or TTH. 
While an array of impairments may present, we contend that musculoskeletal disorders involving joint dysfunction basically present with interrelated dysfunction of the movement and muscle systems. Changes in muscle function are well documented in neck pain disorders, including neck related headache. ${ }^{12} 2526$ The ICHD_3 and the Cervicogenic Headache International Study Group diagnostic criteria, in common, nominate both restricted range of cervical movement and painful segmental joint dysfunction (positive response to anaesthetic blocks) as musculoskeletal criteria for the diagnosis of cervicogenic headache. ${ }^{1427}$ Thus to have certainty in interpretation of cervical musculoskeletal impairment in migraine or TTH, measures of cervical range of movement and joint dysfunction together with a measure of motor output which typify a musculoskeletal disorder should be included in all future studies. This core set of measures has been shown to have high sensitivity and specificity to identify cervicogenic headache, a headache secondary to a cervical musculoskeletal disorder ${ }^{12}$

The presence of interrelated dysfunction in this core set of measures would more convincingly implicate cervical musculoskeletal dysfunction as a cause of neck pain in migraine and TTH or their variants. This does not mean that other tests and measures cannot be undertaken and researchers can add additional tests as is relevant to their research question.

\section{Application and interpretation of clinical tests}

Whether in clinical practice or research, the results of any test or measure are dependent on the quality and accuracy of methodology as well as interpretation of outcomes. The reliability of clinical tests for cervical joint, movement and muscle dysfunction have proven acceptability for use as the core set of musculoskeletal measures. ${ }^{1128-33}$ However the need for skilful manual handling and skilful qualitative evaluation for accurate test results should be appreciated. Likewise care must be taken to consider the clinical significance and relevance of measurement values and not statistical significance only.

\section{Conclusion}

Currently, there is a lack of clarity about the relationship between migraine and TTH associated neck pain and cervical musculoskeletal dysfunction. Dysfunctions in the musculoskeletal system do not occur in isolation. It is proposed that the picture can be made clearer if simultaneous changes in cervical movement, joint and muscle function were core requirements in measures of cervical function to link headache associated neck pain with a musculoskeletal disorder. 


\section{References}

1. Collaborators GDallaP. Global, regional, and national incidence, prevalence, and years lived with disability for 310 diseases and injuries, 1990-2015: a systematic analysis for the Global Burden of Disease Study 2015. The Lancet 2016;388:1545-602.

2. Stovner L, Hagen K, Jensen R, et al. The global burden of headache: a documentation of headache prevalence and disability worldwide. Cephalalgia 2007;27(3):193-210.

3. Bartsch T. Migraine and the Neck: New Insights from Basic Data. Current Pain and Headache Reports 2005;9:191-96.

4. Kaniecki R. Migraine and tension-type headache: an assessment of challenges in diagnosis. Neurology 2002;58(9 Suppl 60):S15-20.

5. Viana M, Sances G, Terrazzino S, et al. When cervical pain is actually migraine: An observational study in 207 patients. Cephalalgia 2018:in press.

6. Luedtke K, Stark W, May A. Musculoskeletal dysfunction in migraine patients. Cephalalgia 2018;38(5):865 - 75.

7. Watson $D$, Drummond P. Head pain referral during examination of the neck in migraine and tensiontype headache. Headache 2012;52:1226-35.

8. Steiner T, Stovner L, Vos T. GBD 2015: migraine is the third cause of disability in under 50s. J Headache Pain 2016;17(1):104.

9. Fernández-de-las-Peñas $C$, Alonso-Blanco $C$, Cuadrado $M$, et al. Forward head posture and neck mobility in chronic tension-type headache: a blinded, controlled study. Cephalalgia 2006;26(3):314-9.

10. Florencio $L$, Oliveira $A$, Lemos $T$, et al. Patients with chronic, but not episodic, migraine display altered activity of their neck extensor muscles. J Electromyogr Kinesiol 2016 30:66-72.

11. Hall T, Briffa K, Hopper D, et al. Comparative analysis and diagnostic accuracy of the cervical flexion-rotation test. J Headache Pain 2010;11(5):391-7.

12. Jull G, Amiri M, Bullock-Saxton J, et al. Cervical musculoskeletal impairment in frequent intermittent headache. Part 1: Subjects with single headaches. Cephalalgia 2007;27(7):793802.

13. Zwart JA. Neck mobility in different headache disorders. Headache 1997;37:6-11.

14. Society HCSotIH. The International Classification of Headache Disorders 3rd Edition. Cephalalgia 2018;38 (1):1-211.

15. Blumenfeld A, Siavoshi S. The Challenges of Cervicogenic Headache. Curr Pain Headache Rep 2018;22(7):47.

16. Yin Z, Dong Z, Lu X, et al. A clinical decision support system for the diagnosis of probable migraine and probable tension-type headache based on case-based reasoning. J Headache Pain 2015;16:29.

17. Florencio L, Ferracini G, Chaves T, et al. Active Trigger Points in the Cervical Musculature Determine the Altered Activation of Superficial Neck and Extensor Muscles in Women With Migraine. Clin J Pain 2017;33(3):238-45.

18. Palacios-Ceña $M$, Castaldo $M$, Wang $K$, et al. Relationship of active trigger points with related disability and anxiety in people with tension-type headache. Medicine 2017 96(13):e6548.

19. Anderson R, Sawyer T, Wise D, et al. Painful Myofascial Trigger Points and Pain Sites in Men With Chronic Prostatitis/Chronic Pelvic Pain Syndrome. Journal of Urology 2009;182(6):2753-58.

20. Stratton P, Khachikyan I, Sinaii N, et al. Association of chronic pelvic pain and endometriosis with signs of sensitization and myofascial pain. Obstetrics \& Gynecology 2015;125(5):719-28.

21. Luedtke K, Boissonnault W, Caspersen N, et al. International consensus on the most useful physical examination tests used by physiotherapists for patients with headache: A Delphi study. Man Ther 2016 23(17-24). 
22. Stenneberg M, Rood M, Bie Rd, et al. To what degree does active cervical range of motion differ between patients with neck pain, patients with whiplash, and those without neck pain? A systematic review and meta-analysis. Arch Phys Med Rehabil 2017;98(7):1407-34.

23. Niederer D, Vogt L, Wilke J, et al. Age-related cutoffs for cervical movement behaviour to distinguish chronic idiopathic neck pain patients from unimpaired subjects. Eur Spine J 2015 24(3):493-502.

24. Schneider G, Jull G, Thomas K, et al. Derivation of a clinical decision guide in the diagnosis of cervical facet joint pain. Arch Phys Med Rehabil 2014;95(9):1695-701.

25. Falla DL, Jull GA, Hodges PW. Patients with neck pain demonstrate reduced electromyographic activity of the deep cervical flexor muscles during performance of the craniocervical flexion test. Spine 2004;29(19):2108-14.

26. Watson $\mathrm{DH}$, Trott PH. Cervical headache: an investigation of natural head posture and upper cervical flexor muscle performance. Cephalalgia 1993;13(4):272-84.

27. Sjaastad O, Fredriksen TA, Pfaffenrath V. Cervicogenic headache: diagnostic criteria. The Cervicogenic Headache International Study Group. Headache 1998;38:442-45.

28. Hall T, Briffa K, Hopper D, et al. Long-term stability and minimal detectable change of the cervical flexion-rotation test. J Orthop Sports Phys Ther 2010;40(4):225-9.

29. Hall T, Briffa K, Hopper D, et al. Reliability of manual examination and frequency of symptomatic cervical motion segment dysfunction in cervicogenic headache. Man Ther 2010;15(6):542-6.

30. Jonsson A, Rasmussen-Barr E. Intra- and inter-rater reliability of movement and palpation tests in patients with neck pain: A systematic review. Physiother Theory Pract 2018;34(3):165-80.

31. Jørgensen R, Ris I, Falla D, et al. Reliability, construct and discriminative validity of clinical testing in subjects with and without chronic neck pain. BMC Musculoskelet Disord 2014 4(15):408.

32. Jull G, Treleaven J, Versace G. Manual examination: is pain provocation a major cue for spinal dysfunction? Aust J Physiother 1994;40(3):159-65.

33. Schneider G, Jull G, Thomas K, et al. Intrarater and interrater reliability for select clinical tests in patients referred for diagnostic facet joint blocks in the cervical spine. Arch Phys Med Rehab 2013;94:1628-34. 